\title{
Investigação neurorradiológica de pacientes com deficiência idiopática de hormônio do crescimento
}

\author{
Neuroradiological investigation in patients with idiopathic growth hormone deficiency \\ Maria Alice N. Bordallo1, Leandro D. Tellerman², Rodrigo Bosignoli ${ }^{3}$, Fernando F. R. M. Oliveira ${ }^{3}$,
Fernanda M. Gazolla4, Isabel R. Madeira5, José Fernando C. Zanier6, Jodélia L. M. Henriques
}

\section{Resumo}

Objetivo: Avaliar a freqüência e os tipos de alterações observadas à tomografia computadorizada e ressonância magnética em pacientes com deficiência aparentemente idiopática de hormônio do crescimento e investigar a possível relação entre imagem neurorradiológica e presença de deficiência isolada e múltipla de hormônio do crescimento.

Métodos: Realizamos tomografia computadorizada e ressonância magnética da região hipotálamo-hipofisária em 37 pacientes com deficiência de hormônio do crescimento. Os pacientes foram divididos em deficiência isolada de hormônio do crescimento (Grupo A) e deficiência múltipla de hormônio do crescimento (Grupo B).

Resultados: A tomografia computadorizada foi normal em 25 (68\%) e alterada em $12(32 \%)$ pacientes. Observamos sela vazia em $50 \%$ dos pacientes, parcialmente vazia em $17 \%$ e hipoplasia hipofisária em $33 \%$. Não observamos diferença entre o percentual de alterações à tomografia computadorizada entre os Grupos A e B $(p=0,55)$. A ressonância magnética foi normal em 17 (46\%) e alterada em 20 (54\%) pacientes. À ressonância magnética, observamos sela vazia em $10 \%$, parcialmente vazia em $15 \%$ e hipoplasia hipofisária em $75 \%$ dos pacientes. Entre os pacientes com ressonância magnética alterada, $70 \%$ apresentavam neuro-hipófise ectópica, e em $60 \%$ a haste hipofisária estava afilada ou ausente. Os pacientes do Grupo B apresentaram maior percentual de alterações à ressonância magnética quando comparados aos do Grupo A $(p=0,03)$. Houve discordância entre tomografia computadorizada e ressonância magnética em $43 \%$; entretanto, não observamos diferença no percentual de anormalidades quando comparamos tomografia computadorizada e ressonância magnética ( $\mathrm{p}=$ $0,06)$.

Conclusão: A hipoplasia hipofisária e a neuro-hipófise ectópica são as alterações mais encontradas em pacientes com deficiência de hormônio do crescimento. A associação de hipoplasia hipofisária com outras anormalidades observadas à ressonância magnética pode sugerir a presença de deficiência múltipla de hormônio do crescimento.

J Pediatr (Rio J). 2004;80(3):223-8: Deficiência de hormônio do crescimento, ressonância magnética, tomografia computadorizada, neuro-hipófise ectópica, hipoplasia hipofisária.

\begin{abstract}
Objective: The aim of this study was to analyze the type and frequency of cranial computed tomography and magnetic resonance imaging anomalies in patients with idiopathic growth hormone deficiency (GHD), and also to investigate the possible relationship between neuroradiological images and the presence of isolated $\mathrm{GH}$ or multiple pituitary hormone deficiency.
\end{abstract}

Methods: Magnetic resonance and computed tomography images were obtained for 37 patients with idiopathic growth hormone deficiency (GHD). The patients were divided into two groups: patients with isolated GH (group A) and patients with multiple pituitary hormone deficiencies (group B).

Results: Computed tomography was normal in $25(68 \%)$, and abnormal in $12(32 \%)$ patients. We observed empty sella in $50 \%$, partially empty sella in $17 \%$ and anterior pituitary hypoplasia in $33 \%$ patients. MRI studies revealed normal findings in the hypothalamuspituitary area in 17 (46\%) and abnormal in $20(54 \%)$ patients. We didn't observed differences in the frequency of computed tomography alterations when groups $A$ and $B$ were compared $(p=0.55)$. With magnetic resonance imaging we observed, empty sella in $10 \%$, partially empty sella in $15 \%$ and anterior pituitary hypoplasia in $75 \%$ patients. Among those patients whose magnetic resonance images were altered, the posterior lobe of the pituitary gland was identified in an abnormal position in $70 \%$, and the hypophyseal stalk was thin or interrupted in $60 \%$. The patients from group B presented a higher frequency of magnetic resonance imaging anomalies $(90 \%)$ when compared to group $\mathrm{A}(10 \%), \mathrm{p}=0.03$. There was disagreement between the two methods in $43 \%$ of cases, but we didn't observe a difference in the frequency of alterations when computed tomography was compared with magnetic resonance imaging $(p=0.06)$.

Conclusions: The most frequent defects observed using magnetic resonance imaging are anterior pituitary hypoplasia and ectopic posterior pituitary lobe. The association of glandular hypoplasia with other magnetic resonance imaging abnormalities can suggest the presence of multiple anterior pituitary deficiencies.

J Pediatr (Rio J). 2004;80(3):223-8: Growth hormone deficiency, magnetic resonance images, cranial computed tomography, hypoplastic anterior pituitary, ectopic posterior pituitary.

\footnotetext{
1. Professora adjunta, Unidade Docente Assistencial de Endocrinologia, Hospital Universitário Pedro Ernesto, Faculdade de Ciências Médicas, Universidade do Estado do Rio de Janeiro (HUPE-UERJ). Coordenadora do Setor de Endocrinologia Pediátrica, HUPE-UERJ, Rio de Janeiro, RJ.

2. Ex-interno da Unidade Docente Assistencial de Endocrinologia, HUPE-UERJ, Rio de Janeiro, RJ.

3. Bolsistas UERJ-PIBIC (Programa Institucional de Bolsas de Iniciação Científica), Rio de Janeiro, RJ.

4. Médica da Unidade Docente Assistencial de Endocrinologia, Hospital Universitário Pedro Ernesto, HUPE-UERJ, Rio de Janeiro, RJ

5. Professora assistente de Pediatria, Faculdade de Ciências Médicas, e da Unidade Docente Assistencial de Endocrinologia, HUPE-UERJ, Rio de Janeiro, RJ.

6. Professor adjunto de Radiologia, Unidade Docente Assistencial de Radiologia, HUPE-UERJ, Rio de Janeiro, RJ.

7. Professora adjunta e chefe da Unidade Docente Assistencial de Endocrinologia, HUPE-UERJ, Rio de Janeiro, RJ.
} 


\section{Introdução}

Desde a virada do século, com o advento da radiografia, o estudo da sela túrcica e da hipófise passou a ser valorizado. A radiografia permaneceu como único método de avaliação durante muitas décadas, até a introdução da tomografia computadoriza (TC), que veio incrementar a capacidade de reconhecer e definir o normal e as variações do patológico. Já nos anos 80 , com o advento da ressonância magnética (RM) e, posteriormente, da angiorressonância, houve um aumento importante na qualidade das imagens obtidas, colocando as técnicas de pneumoencefalografia e angiografia em segundo plano.

Com as técnicas de TC e RM, tem sido possível uma melhor delimitação das estruturas da região hipotálamohipofisária e, conseqüentemente, uma melhor detecção de anormalidades anatômicas indicativas de deficiência de hormônio do crescimento (DGH). Em crianças com baixa estatura, a investigação e o diagnóstico da DGH é, por vezes, problemático, já que diversas causas podem estar envolvidas na etiopatogenia dessa deficiência. Nesses casos, as imagens obtidas por TC e/ou RM facilitam a detecção de anormalidades anatômicas que, na maioria das vezes, são indicadoras de deficiência permanente de hormônio do crescimento $(\mathrm{GH})^{1-5}$.

A RM é um método não-invasivo, de maior sensibilidade em comparação com a TC na identificação de anormalidades na região hipotálamo-hipofisária ${ }^{5}$, e tem sido considerada útil não apenas no diagnóstico mas também na decisão terapêutica de pacientes com DGH.

Anteriormente, a maioria dos casos de DGH era diagnosticada como idiopática; com advento da RM, tem sido possível observar, nesses pacientes, uma anormalidade estrutural característica denominada de síndrome de interrupção da haste hipofisária $3,4,6,7$, a qual, pela TC, é mais difícil de ser identificada ${ }^{5}$. Várias publicações ${ }^{1,3,4,6,8}$ tentam correlacionar as alterações morfológicas visualizadas à RM com a deficiência isolada de hormônio do crescimento (DGHI) ou com deficiência de múltiplos hormônios hipofisários (DGHM). Estudos têm mostrado que crianças com DGHM apresentam maior freqüência de alterações à RM quando comparadas com as que têm $\mathrm{DGHI}^{3,9}$. A síndrome de interrupção da haste hipofisária tem sido associada a DGHM e, portanto, a maior severidade do hipopituitarismo, enquanto que a integridade da haste tem sido observada com maior freqüência em pacientes com DGHI ${ }^{1,4,8}$.

Neste estudo, formulamos a hipótese de que as alterações estruturais da região hipotálamo-hipofisária seriam freqüentes no nosso grupo de pacientes com DGH e de que a presença de anormalidades à RM também poderia ser um indicador da severidade do hipopituitarismo. Como a presença de ectopia de neuro-hipófise é observada freqüentemente em pacientes com $\mathrm{DGH}^{4,6,7}$, imaginamos que este achado, quando presente nos nossos pacientes com baixa estatura, permitiria diagnosticar casos duvidosos, evitando, desta forma, uma investigação laboratorial exaustiva desnecessária.

Este estudo tem, portanto, como objetivos analisar a freqüência e os tipos de alterações observadas à TC e RM em pacientes com deficiência aparentemente idiopática de $\mathrm{GH}$ e investigar a possível relação entre a freqüência de alterações à RM e a severidade do hipopituitarismo.

\section{Métodos}

No período de 1996 a 2003, foram matriculados no Hospital Universitário Pedro Ernesto 64 pacientes com suspeita de DGH. Todos os pacientes foram submetidos à investigação neurorradiológica da região hipotálamo-hipofisária através da realização de TC como parte do protocolo de investigação.

Em estudo retrospectivo observacional, baseado em revisão de prontuários, foram avaliados 37 pacientes que haviam sido submetidos inicialmente a TC e posteriormente a RM da sela túrcica. O intervalo entre a realização da TC e da RM variou de 10,2 $\pm 4,1$ meses.

Os critérios utilizados para diagnóstico de DGH foram: baixa estatura (estatura abaixo do $3^{\circ}$ percentil na curva de Tanner e Whitehouse); velocidade de crescimento inferior a $4 \mathrm{~cm} /$ ano; atraso da idade óssea (> 2 DP abaixo da idade cronológica, de acordo com o método de Greulich e Pyle); pico de $\mathrm{GH}$ inferior a $7 \mathrm{ng} / \mathrm{ml}$ em pacientes impúberes e menor que $10 \mathrm{ng} / \mathrm{ml}$ em pacientes na puberdade em dois testes farmacológicos (insulina e clonidina); presença de anormalidades neurorradiológicas, tais como hipoplasia hipofisária, sela vazia ou síndrome de interrupção da haste hipofisária demonstrada por TC ou RM.

Com o intuito de relacionar a freqüência de alterações estruturais da região hipotálamo-hipofisária com a gravidade do hipopituitarismo, os pacientes foram divididos em dois grupos: Grupo A, aqueles com DGHI; e Grupo B, DGHM.

A TC foi realizada utilizando-se aparelho helicoidal marca GE, modelo Hi Speed, e a RM foi realizada com aparelho Toshiba de 1,5 Tesla. O protocolo selecionado para o estudo da sela túrcica foi de cortes no plano coronal antes e após a utilização do meio de contraste venoso, com espessura de $2 \mathrm{~mm}$ na TC e na RM. Foram obtidas imagens à $R M$ com seqüências em ponderação $T 1$, incluindo o estudo dinâmico após contraste com gadolínio. Na RM, também foram realizados cortes complementares com seqüências ponderadas em T1 no plano sagital e, em alguns casos, seqüências com ponderação em T2 para melhor visualização do espaço liquórico.

Foram observadas alterações morfológicas e estruturais da região hipotálamo-hipofisária, incluindo tamanho e morfologia do lobo anterior da hipófise, localização do lobo posterior, características da haste hipofisária e da região hipotalâmica e estruturas da linha média. A haste hipofisária foi descrita como normal, fina ou ausente. Definimos como sela vazia a invaginação liquórica sem identificação de tecido hipofisário, e como sela parcialmente vazia a existência de liquor na cavidade selar, com visualização de tecido hipofisário. A altura máxima da hipófise foi calculada através de medida perpendicular da maior distância entre a base e o topo da glândula; consideramos como hipoplasia hipofisária casos em que a altura da glândula foi menor que -2 DP quando comparados com controles normais ${ }^{10}$. 
Os exames de neuroimagem foram realizados no Hospital Universitário Pedro Ernesto e avaliados pelo mesmo radiologista, que não tinha conhecimento prévio do grupo em que o paciente se enquadrava ( $A$ ou $B$ ). Todos os exames foram revisados pelo chefe da Disciplina de Radiologia da Faculdade de Ciências Médicas da Universidade do Estado do Rio de Janeiro.

Os dados foram analisados no programa Epi-Info versão 6.04b. Foram utilizados os testes qui-quadrado, exato de Fisher e Kruskal-Wallis.

\section{Resultados}

No presente trabalho, 37 pacientes com diagnóstico de DGH aparentemente idiopática realizaram inicialmente TC e posteriormente RM da região hipotálamo-hipofisária. Os pacientes foram divididos em dois grupos: Grupo A, aqueles com DGHI; e Grupo B, aqueles com DGHM. Os dados clínicos, número de pacientes em cada grupo, média e desvio padrão do escore $z$ da estatura e do pico do $\mathrm{GH}$ após estímulo com clonidina e/ou insulina se encontram na Tabela 1.

Tabela 1 - Número de pacientes em cada grupo, análise estatística do escore $z$ da estatura inicial e do pico de hormônio do crescimento após estímulo (média \pm desvio padrão)

\begin{tabular}{lccc}
\hline & $\begin{array}{c}\text { Grupo A } \\
\text { (DGHI*) }\end{array}$ & $\begin{array}{c}\text { Grupo B } \\
\left(\mathbf{D G H M}^{+}\right)\end{array}$ & $\mathbf{p}^{\ddagger}$ \\
\hline $\begin{array}{l}\text { Número de pacientes } \\
\text { Escore z da estatura }\end{array}$ & $-3,7 \pm 0,8$ & $-4,7 \pm 1,7$ & 0,1 \\
$\begin{array}{l}\text { Pico do hormônio do } \\
\text { crescimento após } \\
\text { estímulo § }\end{array}$ & $2,2 \pm 1,9$ & $1,4 \pm 2,2$ & 0,7 \\
\hline
\end{tabular}

* $\mathrm{DGHI}=$ deficiência isolada de hormônio do crescimento.

† DGHM = deficiência de múltiplos hormônios hipofisários.

$\mp$ Significante $p \leq 0,05$.

$\S$ Clonidina ou insulina.

Trinta pacientes eram impúberes, e sete já haviam iniciado a puberdade na época do estudo (Tanner II). O pico do GH após estímulo com clonidina ou insulina (ITT) foi menor que $10 \mathrm{ng} / \mathrm{ml}$ em todos os pacientes que já estavam na puberdade e menor que $7 \mathrm{ng} / \mathrm{ml}$ nos que eram impúberes, exceto em um paciente impúbere, que apresentou pico de $\mathrm{GH}$ de $8,5 \mathrm{ng} / \mathrm{ml}$ após ITT.

A TC foi normal em 25 (68\%) pacientes e alterada em 12 (32\%). A RM foi normal em 17 (46\%) pacientes e alterada em 20 (54\%). Não observamos diferença significativa na freqüência de alterações na região hipofisária quando comparamos TC e RM nos pacientes com DGH $(p=0,06)$. A comparação dos achados à RM e à TC em pacientes com
DGH idiopática se encontra na Tabela 2. Não observamos diferença significativa na freqüência de alterações na região hipofisária visualizadas à TC entre os pacientes dos Grupos A e $B(p=0,55)$; entretanto, os pacientes do Grupo B apresentaram maior percentual de alterações na região hipotálamo-hipofisária visualizadas à RM quando comparados aos pacientes do Grupo A $(p=0,01)$, como se pode observar na Tabela 3.

Tabela 2 - Comparação dos achados à ressonância magnética (RM) e tomografia computadorizada (TC) em pacientes com deficiência idiopática de hormônio do crescimento

\begin{tabular}{lcc}
\hline & $\begin{array}{c}\text { TC } \\
\mathbf{n}(\%)\end{array}$ & $\begin{array}{c}\text { RM } \\
\mathbf{n}(\%)\end{array}$ \\
\hline Normal & $25(68)$ & $17(46)$ \\
Alterada & $12(32)$ & $20(54)$ \\
Sela vazia & $6(50)$ & $2(10)$ \\
Sela parcialmente vazia & $2(17)$ & $3(15)$ \\
Adeno-hipófise hipoplásica & $4(33)$ & $1(5)$ \\
$\begin{array}{l}\text { Adeno-hipófise hipoplásica }+ \\
\text { Neuro-hipófise ectópica }\end{array}$ & 0 & $14(70)$ \\
\hline
\end{tabular}

Tabela 3 - Análise estatística da comparação do percentual de anormalidades à TC e à RM nos pacientes do Grupo $A$ (DGHI*) e do Grupo B $\left(\mathrm{DGHM}^{+}\right)$

\begin{tabular}{lccc}
\hline & Grupo A & Grupo B & $\mathbf{p}^{\ddagger}$ \\
\hline TC normal & $8(32 \%)$ & $17(68 \%)$ & 0,55 \\
TC alterada & $2(17 \%)$ & $10(83 \%)$ & \\
RM normal & $8(47 \%)$ & $2(10 \%)$ & 0,01 \\
RM alterada & $9(53 \%)$ & $18(90 \%)$ &
\end{tabular}

* DGHI = deficiência isolada de hormônio do crescimento.

† DGHM = deficiência de múltiplos hormônios hipofisários.

$\ddagger$ Significante $p \leq 0,05$
A neuro-hipófise se encontrava em posição ectópica em dois (20\%) pacientes do Grupo A e em 12 (44\%) do Grupo B. A haste hipofisária se encontrava afilada ou ausente em dois (20\%) pacientes do Grupo A e em 10 (37\%) do Grupo B. Não observamos diferença entre o percentual de neurohipófise ectópica e de alterações na haste hipofisária entre os dois grupos ( $p=0,3$ e $p=0,6$ ). A associação entre hipoplasia da adeno-hipófise e ectopia da neuro-hipófise foi observada em $38 \%$ dos pacientes; neuro-hipófise ectópica foi observada apenas à RM e foi o achado mais freqüente $(70 \%)$ entre todas as alterações.

Houve discordância entre os dois métodos (TC e RM) em $16(43 \%)$ pacientes. Em $10(62,5 \%)$ pacientes, a TC 
inicialmente foi normal, e a RM feita posteriormente mostrou hipoplasia da adeno-hipófise associada a ectopia da neuro-hipófise; em quatro pacientes, a TC mostrava sela vazia e a RM não confirmou o diagnóstico, mostrando importante redução do volume da adeno-hipófise em dois casos e volume normal nos outros dois; um paciente apresentava hipoplasia da adeno-hipófise na TC e a RM foi normal; e um paciente com TC normal mostrou sela parcialmente vazia à RM.

\section{Discussão}

Em pacientes com baixa estatura e suspeita de DGH, o estudo neurorradiológico por TC e/ou RM da região hipotálamo-hipofisária tem possibilitado identificar lesões destrutivas e alterações estruturais anatômicas que, na maioria das vezes, são indicadoras de deficiência permanente de $\mathrm{GH}^{1-5}$. Em algumas situações, como observado nas deficiências hormonais parciais, o diagnóstico de DGH pode ser difícil, sendo necessária uma investigação laboratorial dispendiosa, complexa e, muitas vezes, de difícil interpretação. As imagens obtidas por RM exibem maior grau de acurácia quando comparadas com as obtidas por TC, sendo a RM, portanto, atualmente considerada um método superior à TC na investigação de pacientes com $\mathrm{DGH}^{5}$.

Estudos recentes em crianças com baixa estatura que sabidamente apresentam DGH de causa não-tumoral mostram que a principal alteração encontrada na RM é a hipoplasia hipofisária associada à ruptura da haste e a ectopia da neuro-hipófise ${ }^{1-4,6}$, sendo esta última dificilmente visualizada por TC ${ }^{5}$. Portanto, é consenso que, na investigação da baixa estatura, a presença à RM de hipopla- sia hipofisária associada à ectopia da neuro-hipófise pode ser um forte indício de $\mathrm{DGH}^{1,3,4,11,12}$. Entre os nossos pacientes, 54\% apresentavam alteração à RM, e entre as anormalidades observadas, a hipoplasia hipofisária associada à neuro-hipófise ectópica foi o achado mais freqüente, estando presente em $70 \%$ dos casos. Os resultados obtidos neste estudo estão em acordo com dados da literatura, que também mostram um grande número de alterações morfológicas na região hipotálamo-hipofisária à RM de pacientes com DGH, sendo freqüente a presença de ectopia da neurohipófise $e^{4,6,9}$. Entre os nossos pacientes com baixa estatura e suspeita de DGH, tivemos um que apresentava TC normal, IGF-1 dentro dos limites mínimos da normalidade (-2 DP), resposta do GH no ITT normal para o estágio puberal, em que a presença de hipoplasia hipofisária à RM, associada à neuro-hipófise ectópica (Figura 1), foi decisiva para a elucidação do diagnóstico de DGH. Outros autores ${ }^{11,12}$ também valorizam as alterações observadas à RM como sendo importantes no diagnóstico diferencial de crianças com baixa estatura, pois, diante das dificuldades de se estabelecer um valor de normalidade e de reprodutibilidade nos testes de estimulação do $\mathrm{GH}$, a presença de anormalidades estruturais da região hipotálamo-hipofisária nessas crianças reforça a possibilidade de deficiência hormonal. No presente estudo, a constatação à RM de hipoplasia hipofisária e neuro-hipófise ectópica também auxiliou no diagnóstico de DGH, principalmente nos pacientes com faixa etária mais jovem, com baixo peso, que tinham dificuldades na realização de provas farmacológicas de estimulação do $\mathrm{GH}$, em que a grande variação dos níveis de IGF-1 decorrente da faixa etária impossibilitava a discriminação entre o normal e o patológico.
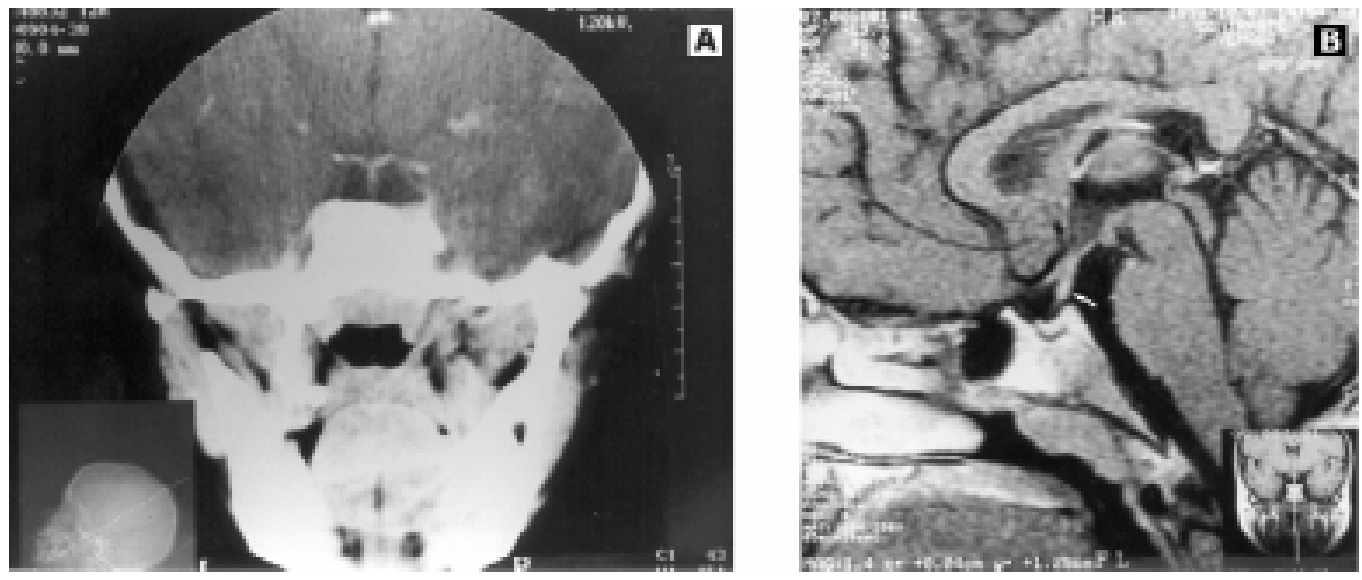

Figura 1 - Paciente apresentando tomografia computadorizada normal (A) e hipoplasia da adeno-hipófise, neuro-hipófise ectópica e ausência de haste hipofisária à ressonância magnética (B) 
Como crítica ao nosso estudo, podemos citar o fato de que os exames foram realizados em épocas diferentes, 0 que pode ter colaborado com a discordância entre os resultados da TC e da RM. Entretanto, em pacientes com DGH, têm sido descritas modificações do volume selar durante a evolução da doença ${ }^{13}$. Assim, não podemos descartar a possibilidade de que, ao longo do acompanhamento, nossos pacientes tenham apresentado modificações do volume hipofisário em decorrência da evolução normal do hipopituitarismo. Essa hipótese explicaria a discordância entre os dois métodos, encontrada em $46 \%$ dos pacientes, reforçando a idéia de que as diferenças entre os achados na TC e na RM seriam decorrentes da evolução natural da doença, e não inerentes ao método de investigação utilizado. Na nossa casuística, dos 16 pacientes em que o laudo da TC foi discordante do laudo da RM, 10 (62,5\%) apresentavam TC normal e RM alterada (hipoplasia hipofisária e neuro-hipófise ectópica). Talvez um dos motivos para uma discordância tão alta entre os resultados obtidos pelos dois métodos tenha sido o fato da ectopia da neuro-hipófise dificilmente ser diagnosticada por TC. E, como as imagens por RM exibem maior grau de acurácia em identificar o tecido hipofisário ${ }^{5}$, encontramos entre os nossos pacientes variações nas medidas da adeno-hipófise na comparação da TC com a RM. Uma outra vantagem da RM está no fato de as imagens serem adquiridas de forma multiplanar, diferentemente da TC, em que são feitas reconstruções sagitais das imagens, o que pode levar a confusões no diagnóstico, principalmente quando são utilizados tomógrafos não-helicoidais e cortes com maior espessura.

No nosso estudo, não observamos diferença estatística entre a prevalência de anormalidades observadas na região hipotálamo-hipofisária quando comparamos TC (32\%) e RM (54\%). Entretanto, concordamos que, ao comparar os dois métodos, a RM, por ser um método mais sensível para a avaliação do tecido hipofisário, parece ser superior no estudo da região hipotálamo-hipofisária de pacientes com $D_{G H}^{5}$. O fato dos nossos pacientes com DGHM terem apresentado maior freqüência de alterações à RM quando comparados com pacientes com DGH confirma nossa hipótese de que a presença de anormalidades à RM poderia ser um indicador da severidade do hipopituitarismo. Entretanto, em discordância com outros estudos ${ }^{1,4}, 8$, não observamos relação entre ausência da haste hipofisária e/ou haste afilada à RM e pan-hipopituitarismo.

Sabendo que a DGH é freqüentemente associada à secreção anormal de outros hormônios hipofisários, achamos importante que pacientes com DGHI que apresentem anormalidades à RM sejam submetidos periodicamente a avaliação hormonal, uma vez que eles podem evoluir, ao longo do curso da sua doença, para falência de múltiplos hormônios hipofisários. Diante dos resultados obtidos neste estudo, podemos imaginar que alguns dos nossos pacientes com DGHI e haste hipofisária afilada ou não visualizada à RM possam também evoluir, com o tempo, para deficiência de outras trofinas hipofisárias não detectadas numa avali-

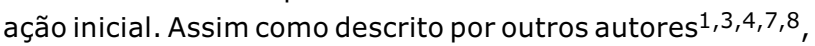
também não observamos diabetes insípido nos nossos pacientes que apresentavam ectopia da neuro-hipófise.
Estudos recentes ${ }^{13,14}$ têm mostrado que indivíduos com pan-hipopituitarismo congênito causado por mutações no gene PROP-1 podem apresentar alterações na região hipotálamo-hipofisária possíveis de serem visualizadas pela RM, sugerindo que o PROP-1 tenha um papel crucial na organogênese e na diferenciação da adenohipófise ${ }^{14}$. Também tem sido demonstrado que pacientes portadores de mutações genéticas do PROP-1, do GHRH-R e do GH-1 apresentam maior freqüência de integridade da haste hipofisária e de localização normal da neuro-hipófise quando comparados a pacientes com DGH sem mutação15,16. Não foi possível realizar estudo genético nos nossos pacientes, mas acreditamos que aqueles com DGH que apresentam haste hipofisária íntegra e neuro-hipófise de localização normal à RM mereçam ser melhor investigados quanto a possíveis mutações genéticas.

Pelos resultados obtidos neste estudo, concluímos que a presença de alterações estruturais da região hipotálamohipofisária em pacientes com DGH sugere maior gravidade do hipopituitarismo, podendo o estudo neurorradiológico do eixo hipotálamo-hipofisário por RM não apenas ajudar no diagnóstico de pacientes com suspeita de DGH, mas também auxiliar na seleção dos candidatos para o estudo de possíveis mutações genéticas, contribuindo, desta forma, para a elucidação da etiologia da doença.

\section{Referências}

1. Arrigo T, De Luca F, Maghnie M, Blandino A, Lombardo $F$, Messina MF, et al. Relationships between neuroradiological and clinical features in apparently idiopathic hypopituitarism. Europ J Endocrinol. 1998;139:84-8.

2. Barros L, Ribeiro C, Bastos M, Rodrigues D, Moura C, Geraldes $E$, et al. Craniocerebral imaging in children with short stature. Acta Med Port. 1997;10(5):361-5.

3. Bozzolla M, Adamsbaum C, Biscaldi I, Zecca M, Cisternino M, Genovese $E$, et al. Role of magnetic resonance imaging in the diagnosis and prognosis of growth hormone deficiency. Clin Endocrinol. 1996;45:21-6.

4. Hamilton J, Blaser S, Danemen D. MR imaging in the diagnosis and prognosis of growth hormone deficiency. Am J Neuroradiol. 1998;19:1609-15.

5. Maghnie M, Triulzi F, Larizza D, Scotti G, Cecchini A, Severi F. Hypotalamic-pituitary dwarfism: comparison between MR imaging and CT findings. Pediatr Radiol. 1990;20(4):229-35.

6. Tillman V, Tang VW, Price DA, Hughes DG, Wright NB, Clayton PE. Magnetic resonance imaging of the hypotalamic-pituitary axis in the diagnosis of growth hormone deficiency. J Pediatr Endocrinol Metab. 2000;13(9):1577-83.

7. Chen S, L'Eger J, Garel C, Hassam M, Czernichow P. Growth hormone deficiency with ectopic neurohypophysis: anatomical variations and relationship between the visibility of the pituitary stalk asserted by magnetic resonance imaging and anterior pituitary function. J Clin Endocrinol Metab. 1999;84:2408-13.

8. Maghnie M, Triulzi F, Larizza D, Preti P, Priora C, Scotti G. Hypothalamic-pituitary dysfunction in growth-hormone-deficient patients with pituitary abnormalities. J Clin Endocrinol Metab. $1991 ; 73: 79-83$.

9. Truilzi F, Scotti G, di Natale B, Pellini C, Lukezic M, Scognamiglio $M$. Evidence of a congenital midline brain anomaly in pituitary dwarfs: a magnetic resonance imaging study in 101 patients. Pediatrics. 1994;93:409-16.

10. Argyropoulou M, Perignon F, Brunelle F, Brauner R, Rappaport $R$. Height of normal pituitary gland as a function of age evaluated by magnetic resonance imaging in children. Pediatr Radiol. 1991;21:247-9.

11. Argyropoulou M, Perignon F, Brauner R, Brunelle F. Magnetic resonance imaging in the diagnosis of growth hormone deficiency. J Pediatr. 1992;120:886-91. 
12. Drummond JB, Martins JCT, Soares MMS, Dias EP. Alterações da haste hipofisária e suas implicações clínicas. Arq Bras Endocrinol Metab. 2003;47(4):458-66.

13. Teinturier C, Vallet S, Adamsbaum C, Bendaoud M, Brue T, Bougnères PF. Pseudotumor of pituitary due to Prop-1 deletion. J Pediatr Endocrinol Metab. 2002;15:95-101.

14. Fofanova O, Takamura N, Kinoshita Ei-ichi, Vorontsov A, Vladimirova $\mathrm{V}$, et al. MR imaging of the pituitary gland in children and young adults with congenital combined pituitary hormone deficiency associated with PROP I mutations. Am J Roentgenol. 2000;174:555-9.

15. Osório MGF, Marui S, Jorge AAL, Latronico AC, Lo LSS, Leite CC, et al. Pituitary magnetic resonance imaging and function in patients with growth hormone deficiency with and without mutations in GHRH, GH-1, or PROP-1 genes. J Clin Endocrinol Metab. 2002;87(11):5076-84.
16. Sung-Su K, Youngho K, Young-Lim S, Gu-Hwan K, Tae-Ue K, Han-Wook Y. Clinical characteristics and molecular analysis of $P I T 1, P R O P 1, L H X 3$, and HESX1 in combined pituitary hormone deficiency patients with abnormal pituitary MR imaging. Horm Res. 2003;60(6):277-83.

Correspondência:

Maria Alice Neves Bordallo

Rua Praia de Botafogo, 132/501

CEP 22250-040 - Rio de Janeiro, RJ

Tel.: (21) 2551.4986

Fax: (21) 2551.4986/2553.5553

E-mail: maliceb@attglobal.net 